\title{
Reactions of $\boldsymbol{N}$-acylhydrazones with thionyl chloride
}

\author{
Antigoni Kotali* and Ioannis S. Lafazanis \\ Laboratory of Organic Chemistry, College of Engineering, University of Thessaloniki, \\ Thessaloniki, GR-54006, Greece \\ E-mail: kotali@eng.auth.gr
}

\section{Dedicated to Professor A. Varvoglis on the occasion of his $65^{\text {th }}$ birthday}

(received 3 Feb 03; accepted 2 May 03; published on the web 06 May 03)

\begin{abstract}
$\mathrm{N}$-Acylhydrazones of $\mathrm{o}$-hydroxyaryl ketones react with thionyl chloride to give $\mathrm{N}$ arylhydrazonoyl chlorides in good yields.
\end{abstract}

Keywords: $N$-Acylhydrazones, thionyl chloride, imidoyl chlorides, o-hydroxyaryl ketones, hydrazonoyl chlorides

\section{Introduction}

Imidoyl halides are very useful reagents in organic synthesis. ${ }^{1} \mathrm{~N}$-Arylhydrazonoyl halides are important members of this family. They have attracted a lot of interest in recent years mostly because of their synthetic usefulness. Several reviews presenting their chemistry appeared in the literature. $^{1-4}$ There are also some references about their structure. The presence of $\mathrm{C}=\mathrm{NNH}$ moiety at the molecule gives the possibility of formation of $(E)$ and $(Z)$ isomers. It has been confirmed by spectroscopic studies ${ }^{5,6}$ as well as X-ray diffraction analysis ${ }^{6-8}$ that hydrazonoyl halides exist as $(Z)$ isomers. Furthermore, it is known that $(Z)$ isomers are thermodynamically more stable. ${ }^{9}$

Our continuous interest ${ }^{10-12}$ in the use of $N$-acylhydrazones of aromatic aldehydes and ketones as starting materials for new molecules led us to investigate their reaction with thionyl chloride as a possible route to the synthesis of $N$-arylhydrazonoyl chlorides.

\section{Results and Discussion}

Commercially available salicylaldehyde $\mathbf{1}$ was reacted with hydrazide $\mathbf{2}$, obtained also commercially, to prepare hydrazones $\mathbf{3}$ in very good yields according to the literature method. ${ }^{10}$ 
Reaction of 3 with thionyl chloride in benzene at room temperature yielded $N$-arylhydrazonoyl chlorides 4 in 58-70 \% yield (Scheme 1). The products were identified by their NMR and MS spectra and elemental analysis.

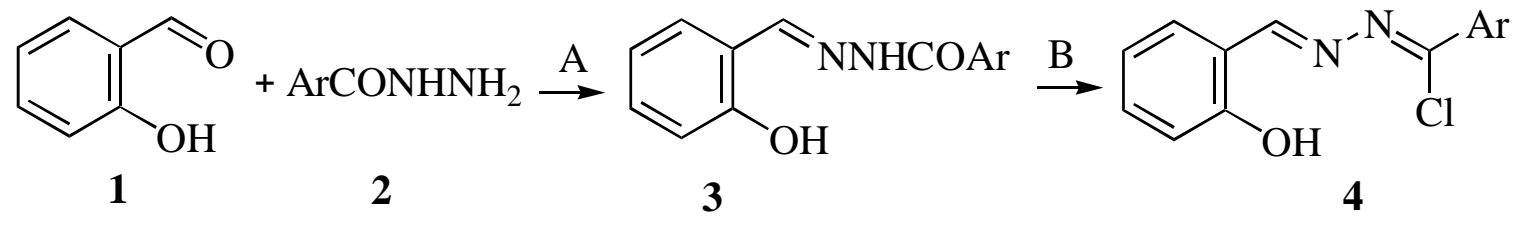

a: $\mathrm{Ar}=\mathrm{Ph} \quad \mathrm{b}: \mathrm{Ar}=\mathrm{O}-\mathrm{HOC}_{6} \mathrm{H}_{4} \quad \mathrm{c}: \mathrm{Ar}=O-\mathrm{O}_{2} \mathrm{NC}_{6} \mathrm{H}_{4}$

A: propanol-2/reflux/24 h/82-90\% B:SOCl $/$ benzene/reflux/ 2 h/ 58-70\%

\section{Scheme 1}

Subsequently, the brominated hydrazone $\mathbf{5}$ was prepared by treatment of salicylic aldehyde benzoyl hydrazone 3a with bromine according to the literature method. ${ }^{10}$ The reaction of $\mathbf{5}$ with thionyl chloride afforded the bis-imidoyl dihalide 6 in good yield (Scheme 2).<smiles>Oc1ccc(Br)cc1C(Cl)=NN=C(Br)c1cc(Br)ccc1O</smiles>

A: $\mathrm{Br}_{2} / \mathrm{AcOH} / \mathrm{NaOAc} / 25^{\circ} \mathrm{C}, 2 \mathrm{~h} / 67 \%$ B: $\mathrm{SOCl}_{2} /$ benzene/reflux, $2 \mathrm{~h} / 70 \%$

\section{Scheme 2}

Furthermore, treatment of 4a with aniline in dimethylformamide led to the formation of derivative 7, in $83 \%$ yield (Scheme 3). The anilino-derivative 7 was formed as a mixture of two isomers as it is indicated by the ${ }^{1} \mathrm{H}$ and ${ }^{13} \mathrm{C}$ NMR spectra.

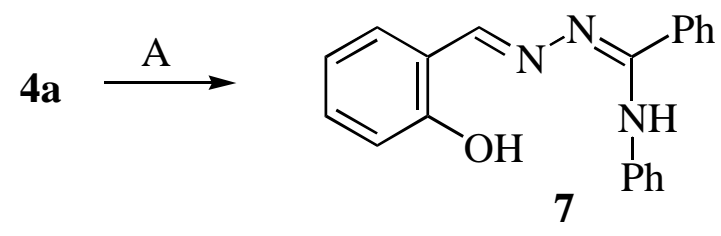

A: $\mathrm{PhNH}_{2} / \mathrm{DMF} / 25^{\circ} \mathrm{C}, 24 \mathrm{~h} / 83 \%$

\section{Scheme 3}


Finally, attempts to perform the thionyl chloride reactions with $N$-benzoylhydrazone of $O$ hydroxy-acetophenone, with $N$-benzoyl hydrazone of $o$-amino-acetophenone as well as with $N$ carbonylethoxy hydrazone of $o$-amino-acetophenone were unsuccessful. They all gave complicated mixtures. In contrast, it is known that $N$-carbonylethoxy hydrazone of acetophenone and o-hydroxy-acetophenone afford 4-phenyl-1,2,3-thiadiazole and 4-(o-hydroxyphenyl)-1,2,3thiadiazole respectively in good yields. ${ }^{13,14}$

\section{Experimental Section}

General Procedures. Melting points were determined using a Kofler apparatus and are uncorrected. ${ }^{1} \mathrm{H}$ NMR (300 MHz) and ${ }^{13} \mathrm{C}$ NMR (75 MHz) spectra were recorded on a Varian Unity Plus spectrometer. MS were measured on a Platform II spectrometer. Thionyl chloride, hydrazine hydrate, ketones and acylhydrazides were all purchased from Aldrich. $N$ Acylhydrazones was prepared according to the literature methods. ${ }^{11,15}$

\section{General procedure for the reaction of hydrazones with thionyl chloride}

Thionyl chloride $(2.5 \mathrm{~mL}$ ) was added dropwise to a solution of $N$-acylhydrazone $\mathbf{3}$ or $\mathbf{5}$ (5 mmol) in benzene $(10 \mathrm{~mL})$. The reaction mixture was refluxed for 2 hours. The solvent was then evaporated and the residue was subjected to column chromatography (silica gel, 70-230 mesh) and it was eluted with petroleum ether/chloroform 5:1 to afford the pure products $\mathbf{4 a - 4 c}$ and $\mathbf{6}$.

$\mathrm{N}$-Salicyliminophenylmethanehydrazonoyl chloride (4a). Yellow solid; mp $122-124{ }^{\circ} \mathrm{C}$; yield 70\%; ${ }^{1} \mathrm{H}$ NMR $\left(\mathrm{CDCl}_{3}\right) \delta$ 6.63-7.09 (m, 2H), 7.39-7.54 (m, 5H), 7.56-8.15 (m, 2H), 8.76 (s, 1H), 12.40 (s, 1H); ${ }^{13} \mathrm{C}$ NMR $\left(\mathrm{DMSO}_{d 6}\right) \delta 117.5,118.7120 .5,129.2,129.7,132.3,133.2,133.6$, 134.8, 159.9, 164.5; MS m/z (EI+): 258 (M), 223; Anal. Calcd for $\mathrm{C}_{14} \mathrm{H}_{11} \mathrm{ClN}_{2} \mathrm{O}$ : C, 64.99; H, 4.28; N, 10.82; Cl, 13.70. Found: C, 64.79; H, 4.28; N, 10.86; Cl, 13.63.

$\mathbf{N}$-Salicylimino-o-hydroxyphenylmethanehydrazonoyl chloride (4b). Yellow semi-solid; yield 65\%; ${ }^{1} \mathrm{H}$ NMR $\left(\mathrm{DMSO}_{d 6}\right) \delta$ 6.87-6.97 (m, 4H), 7.25-7.39 (m, 1H), 7.41-7.44 (m, 1H), 7.527.54 (m, 1H), 7.87-7.89 (m, 1H), 8.67 (s, 1H), 11.8 (br, 1H), 12.05 (s, 1H); ${ }^{13} \mathrm{C} \mathrm{NMR}\left(\mathrm{DMSO}_{d 6}\right)$ $\delta$ 114.0, 114.9, 115.7, 117.1, 117.5, 117.8, 127.1, 127.9, 130.1, 132.4, 147.4, 156.0, 157.5, 163.0; MS m/z (ES+): 274 (M); Anal. Calcd for $\mathrm{C}_{14} \mathrm{H}_{11} \mathrm{ClN}_{2} \mathrm{O}_{2}: \mathrm{C}, 61.21 ; \mathrm{H}, 4.03 ; \mathrm{N}, 10.20$; Cl, 12.90. Found: C, 61.14; H, 4.12; N, 10.26; Cl, 12.75.

$\mathrm{N}$-Salicylimino-o-nitrophenylmethanehydrazonoyl chloride (4c). Yellow semi-solid; yield 58\%; ${ }^{1} \mathrm{H}$ NMR $\left(\mathrm{DMSO}_{d 6}\right)$ $\delta$ 7.30-7.32 (m, 1H), 7.48-7.55 (m, 2H), 7.70-7.96 (m, 4H), 8.10-8.15 (m, 1H), 8.42-8.44 (s, 1H), $12.06(\mathrm{~s}, 1 \mathrm{H}) ;{ }^{13} \mathrm{C} \mathrm{NMR}\left(\mathrm{DMSO}_{d 6}\right) \delta 124.1,125.1,127.3,129.4$, 131.7, 132.8, 133.0, 133.6, 134.5, 134.9, 145.1, 148.2, 162.0; MS m/z (ES+): 303 (M); Anal. Calcd for $\mathrm{C}_{14} \mathrm{H}_{10} \mathrm{ClN}_{3} \mathrm{O}_{3}$ : C, 55.36; H, 3.32; Cl, 11.67; N, 13.84. Found: C, 55.15; H, 3.32; Cl, 11.66; N, 13.79.

$\mathbf{N}$-2-Hydroxy-5-bromophenylbromoimidoyl-phenylmethanehydrazonoyl chloride (6). Yellow solid; mp 165-166 ${ }^{\circ} \mathrm{C}$; yield 70\%; ${ }^{1} \mathrm{H}$ NMR $\left(\mathrm{DMSO}_{d 6}\right) \delta$ 7.52-7.63 (m, 3H), 7.91-7.93 (m, 2H), 
8.04-8.07 (m, 2H), 8.98 (s, 1H), 12.53 (s, 1H); ${ }^{13} \mathrm{C}$ NMR $\left(\mathrm{DMSO}_{d 6}\right) \delta 110.6,111.4,120.0,128.5$, 129.0, 132.3, 132.9, 134.2, 138.0, 148.0, 155.1, 164.1; MS m/z (ES+): 417 (M+ 1); Anal. Calcd for $\mathrm{C}_{14} \mathrm{H}_{9} \mathrm{Br}_{2} \mathrm{ClN}_{2} \mathrm{O}$ : C, 40.37; H, 2.18; N, 6.72. Found: C, 40.22; H, 2.31; N, 6.68.

Procedure for the reaction of 4 a with aniline. A mixture of imidoyl chloride $4 \mathbf{a}(5 \mathrm{mmol})$ and aniline $(5 \mathrm{mmol})$ in dimethylformamide $(10 \mathrm{ml})$ was stirred at room temperature for 24 hours. The white precipitate was filtered and dried in vacuum to afford the pure product 7 as a mixture of two isomers.

[Phenyl-[( $N$-salicylimino)-imino]-methyl]-aniline (7). White solid; yield, 83\%; ${ }^{1} \mathrm{H}$ NMR $\left(\mathrm{DMSO}_{d 6}\right) \delta$ 6.86-7.00 (m, 7H), 7.26-7.73 (m, 15H), 7.91-8.07 (m, 6H), $8.63(\mathrm{~s}, 1 \mathrm{H}), 8.95$ (s, $1 \mathrm{H}), 11.11(\mathrm{~s}, 1 \mathrm{H}), 11.26(\mathrm{~s}, 1 \mathrm{H}), 11.36(\mathrm{~s}, 1 \mathrm{H}), 12.11(\mathrm{~s}, 1 \mathrm{H}) ;{ }^{13} \mathrm{C}$ NMR $\left(\mathrm{DMSO}_{d 6}\right) \delta 117.1$, 117.2, 117.4, 118.6, 119.3, 120.0, 120.3, 120.4, 122.0, 128.3, 129.2, 129.7, 130.2, 131.5, 132.2, 132.6, 133.1, 133.5, 133.9, 134.7, 146.7, 149.0, 158.2, 159.8, 163.5, 164.4; MS m/z (AP+): 316 $(\mathrm{M}+1)$; Anal. Calcd for $\mathrm{C}_{20} \mathrm{H}_{17} \mathrm{~N}_{3} \mathrm{O}$ : C, 76.17; H, 5.43; N, 13.32. Found: C, 76.08; H, 5.70; N, 13.32.

\section{Acknowledgements}

The authors thank Royal Society of Chemistry for financial support of this work.

\section{References}

1. Schawali, A. S. Chem. Rev. 1993, 93, 2731.

2. Brogini, G.; Molteni, G.; Zecchi, G. Heterocycles 1998, 47, 541.

3. Shawali, A. S.; Abdallah, M. A. Adv. Heterocyclic Chem. 1995, 63, 277.

4. Shawali, A. S. J. Heterocyclic Chemistry 1980, 17, 833.

5. Reichardt, C.; Halbritter, K. Chem. Ber. 1973, 106, 1661.

6. Frohberg, P.; Drutkowski, G.; Wagner, C. Eur. J. Org. Chem. 2002, 1654.

7. Hegerty, A. F.; McCormack, M. T.; Hathaway, B. J.; Hulett, L. J. Chem. Soc., Perkin Trans. 2 1977, 9, 1136.

8. Seccombe, R. C.; Kennard, C. H. L. Aust. J. Chem. 1970, 23, 395.

9. Rowe, J. E.; Hegarty, A. F. J. Org. Chem. 1984, 49, 3083.

10. Kotali, A.; Harris, P. A. Org. Prep. Proc. Int. 1994, 26, 155.

11. Kotali, A.; Papapetrou, M.; Dimos, V.; Harris, P. A. Org. Prep. Proc. Int. 1998, 30(2), 177.

12. Kotali, A. Curr. Org. Chem. 2002, 6, 965.

13. Meier, H.; Trickes, G.; Laping, E.; Merkle, U. Chem. Ber. 1980, 113, 183.

14. D’hooge, B.; Smeets, S.; Toppet, S.; Dehaen, W. Chem. Comm. 1997, 1753.

15. Kotali, A.; Tsoungas, P.G. Tetrahedron Lett. 1987, 28, 4321. 